\title{
Emergency ultrasound diagnosis of a left atrial thrombus and diffuse emboli
}

\author{
Anita Datta $\cdot$ Monica Kapoor $\cdot$ Michelle Pearl-Davis • \\ Penelope Chun Lema
}

Received: 10 February 2011/Accepted: 17 May 2011/Published online: 3 June 2011

(C) Springer-Verlag 2011

\begin{abstract}
Introduction A 71-year-old female with history of atrial fibrillation presented to the emergency department (ED) for abdominal pain. A CT scan in the ED showed an abdominal aortic thrombus and diffuse emboli suspicious for a cardiac source.

Methods A bedside ultrasound was performed which confirmed the presence a dilated left atrium with thrombus. The patient was admitted to the hospital and eventually placed on anticoagulation.

Conclusions Atrial fibrillation is a common cause of atrial thrombus. Intracardiac thrombus can be diagnosed by bedside echocardiography in the ED. Emboli can occur as a complication of a thrombus in the atrium. Management may include anticoagulation or surgical intervention.
\end{abstract}

Keywords Echocardiography · Bedside ultrasound · Atrial thrombus $\cdot$ Emboli $\cdot$ Atrial fibrillation

\section{Case report}

A 71-year-old female presented to the emergency department (ED) with diffuse abdominal pain for $7 \mathrm{~h}$. The patient's past medical history was significant for hypertension, hemorrhagic cerebrovascular stroke and atrial fibrillation. Her vital signs were stable. Physical findings included diffuse abdominal tenderness to palpation and

A. Datta $(\bowtie) \cdot$ M. Kapoor - M. Pearl-Davis · P. C. Lema Department of Emergency Medicine, New York Hospital Queens, 56-45 Main Street, Flushing, NY 11355, USA e-mail: arsingla@gmail.com diminished femoral and posterior tibial pulses bilaterally. Significant laboratory values were leukocytosis of $12.5 \mathrm{~K} / \mu \mathrm{L}$ with $85 \%$ neutrophils and a normal lactic acid of $1.9 \mathrm{mmol} / \mathrm{L}$ (normal venous $0.5-2.2 \mathrm{mmol} / \mathrm{L}$ ). All other laboratory values were normal. The electrocardiogram $(\mathrm{EKG})$ showed atrial fibrillation.

Computed tomography (CT) of the abdomen showed a $5 \mathrm{~cm}$ thrombus within the distal abdominal aorta extending to the common iliac arteries. Splenic and left kidney infarcts were also visualized on the CT scan (Fig. 1), raising concern for a cardiac source of multiple emboli. Bedside ultrasound was performed by the emergency physician using a $4 \mathrm{MHz}$ phased array transducer (Zonare, Mountain View, CA, USA). A thrombus in the abdominal aorta was confirmed (Fig. 2). Bedside echocardiography revealed a dilated left atrium with a large thrombus (Figs. 3, 4). Cardiology was emergently consulted due to the bedside echocardiography findings. A transesophageal echo demonstrated a large well-defined, free floating $3.6 \times 5.2 \mathrm{~cm}$ echogenic mass in the left atrium (LA). The patient could not undergo immediate anticoagulation due to a recent hemorrhagic stroke. The patient was transferred to a nursing home on coumadin after a month-long admission.

\section{Conclusions}

Previous studies have demonstrated the utility of bedside echocardiography in the ED. Bedside echocardiography is routinely used in the ED to diagnose pericardial effusion and assess global cardiac activity $[1,2]$.

Four cardiac views are frequently used in emergency ultrasound. The parasternal long (PSL) view includes the right ventricle (RV) anteriorly and LA and left ventricle 


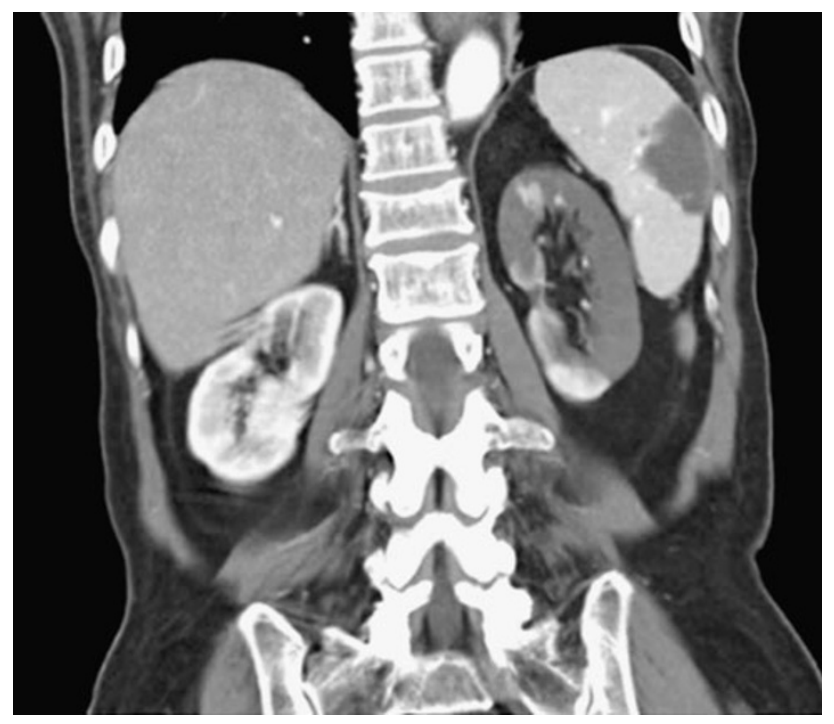

Fig. 1 Abdominal computed tomography demonstrating splenic infarct and left renal infarct

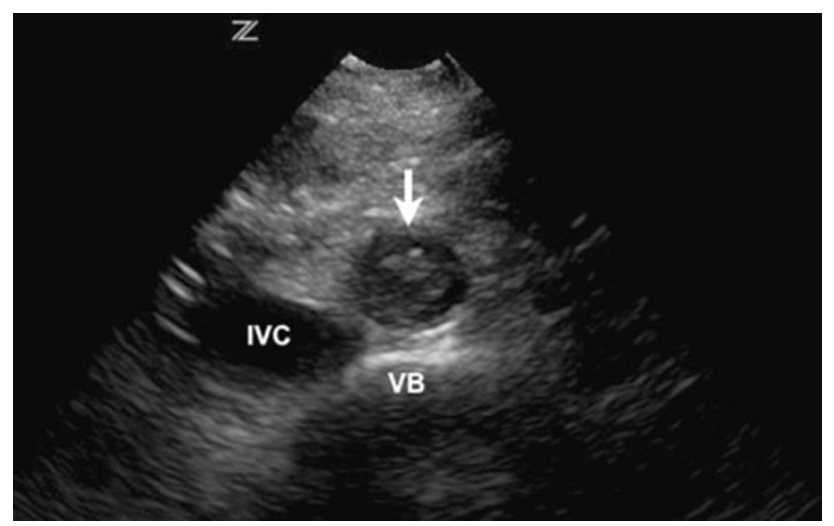

Fig. 2 Transverse view of the middle abdominal aorta with a thrombus (arrow) on bedside ultrasound. IVC inferior vena cava, $V B$ vertabral body

(LV) posteriorly. The right atrium (RA) and the apical region of the LV are not well visualized in the PSL view. The parasternal short (PSS) view is the most useful for analysis of regional ventricular wall motion and global LV contractility. The subxiphoid view is the only satisfactory way to visualize the RA and also affords the best view of the RV. The apical four view is useful for identification of all the chambers and symmetrically displays the mitral and tricuspid valves. This view is most useful for the assessment of the apical region of the LV.

Advanced applications of emergency echocardiography include the assessment of aortic dissection, valvular dysfunction, regional wall motion abnormalities and the identification of intracardiac masses [3]. The differential diagnosis for intracardiac masses include thrombus, myxoma, and metastatic tumor. Thrombus is the most common

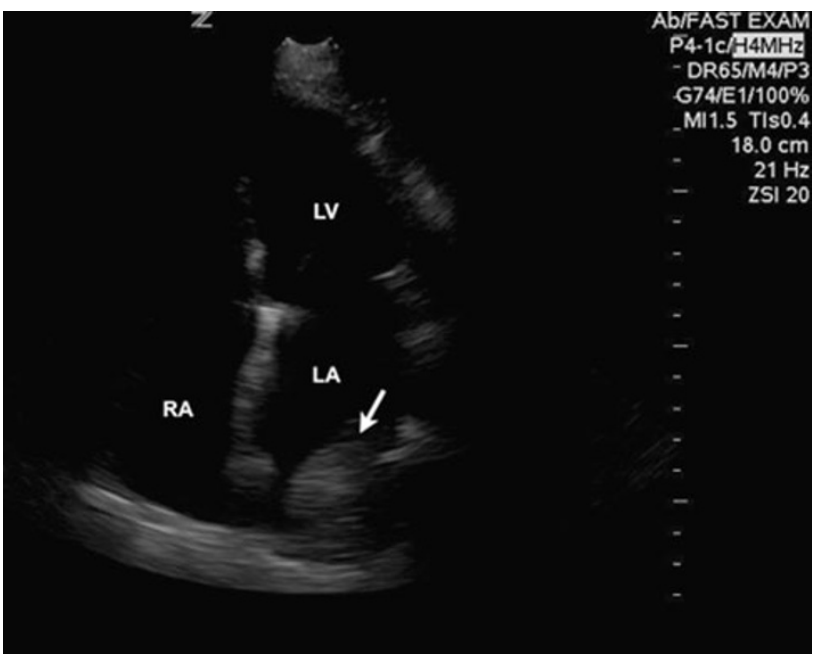

Fig. 3 Bedside echocardiography. Cardiac apical four view with a thrombus (arrow) in left atrium. $L V$ left ventricle, $L A$ left atrium, $R A$ right atrium

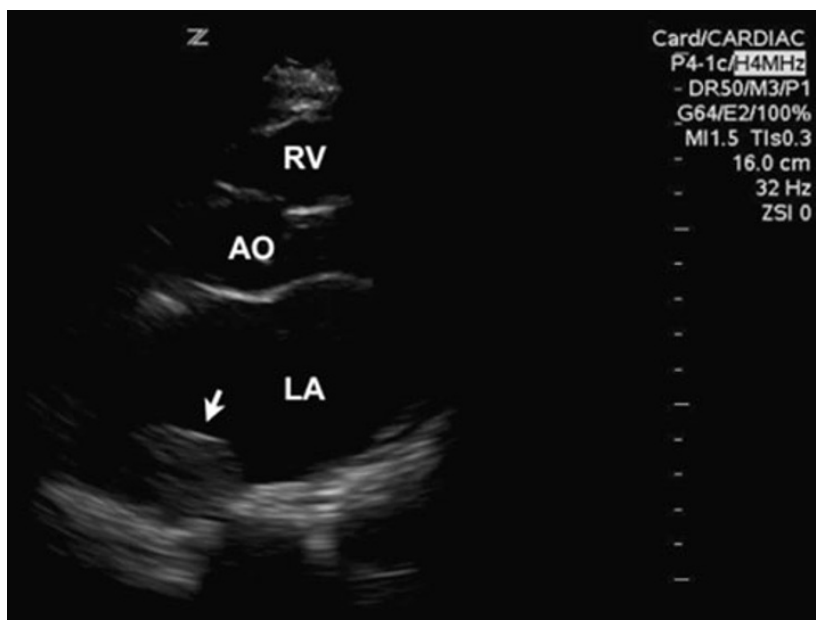

Fig. 4 Bedside echocardiography. Parasternal long view of the heart with a large thrombus (arrow) in a dialated atrium. $L A$ left atrium, $A O$ aortic outflow tract $R V$ right ventricle

cardiac mass. LV thrombi are associated with ventricular aneurysms while LA thrombi are associated with atrial fibrillation [4]. Myxomas should be suspected if the mass is attached to the interatrial septum. Magnetic resonance imaging (MRI) is the diagnostic study of choice to distinguish thrombus from tumor [5].

This case demonstrates the utility of ED physician performed echocardiography to diagnose a dilated left atrium with a thrombus as the source of diffuse emboli. Bedside echocardiography expedited the diagnosis, patient care and treatment.

Conflict of interest None. 


\section{References}

1. Wright J, Jarman R, Connolly J et al (2009) Echocardiography in the emergency department. Emerg Med J 26:82-86

2. Tayal VS, Kline JA (2003) Emergency echocardiography to detect pericardial effusion in patients in PEA and near-PEA states. Resuscitation 59:315-318

3. Labovitz AJ, Noble VE, Bierig M et al (2010) Focused cardiac ultrasound in the emergency setting: a consensus statement of the
American Society of Echocardiography and American College of Emergency Physicians. J Am Soc Echocardiogr 23:1225-1230

4. O'Brien W, O'Brien WT (2009) Top 3 differentials in radiology: a case review. Thiem

5. Carpenter K, Adams D (1998) Apical mural thrombus: technical pitfalls. Heart 80:S6-S8 